\title{
Pacific
}

Journal of

Mathematics

\section{CALCUL DU NOMBRE DE CLASSES DES CORPS DE NOMBRES}

\author{
STÉPHANE LOUBOUTIN
}




\title{
CALCUL DU NOMBRE DE CLASSES DES CORPS DE NOMBRES
}

\author{
STÉPHANE LOUBOUTIN
}

Soit $\mathrm{K} / \mathrm{k}$ une extension de corps de nombres telle que le quotient $\zeta_{K} / \zeta_{k}$ de la fonction zêta de Dedekind $\zeta_{K}$ de $\mathbf{K}$ par celle $\zeta_{k}$ de $\mathbf{k}$ soit holomorphe dans tout le plan complexe (ce qui est par exemple le cas lorsque l'extension $K / k$ est galoisienne). Nous développons un moyen de calcul numérique de la valeur au point 1 de ce quotient $\zeta_{K} / \zeta_{k}$. Une application essentielle de cette évaluation est le calcul numérique du nombre de classes relatif d'un corps $K$ à multiplication complexe, en choisissant pour $\mathrm{k}$ le sous-corps totalement réel maximal de $\mathrm{K}$. En particulier, nous illustrons notre méthode sur l'exemple de corps à multiplication complexe, quartiques et non galoisiens.

1. Notations. Soit $\mathbf{N}$ un corps de nombres de degré $n=r_{1}+2 r_{2}$, où $r_{1}$ désigne bien évidemment le nombre de plongements réels et $2 r_{2}$ le nombre de plongements complexes deux à deux conjugués de $\mathbf{N}$. Nous notons $\zeta_{N}$ sa fonction zêta de Dedekind, $d(N)$ la valeur absolue de son discriminant, $\operatorname{Reg}(N)$ son régulateur et posons

$A_{N}=2^{-r_{2}} d(N)^{1 / 2} \pi^{-\left(r_{1}+2 r_{2}\right) / 2}$

$\lambda_{N}=\frac{2^{r_{1}} h(N) \operatorname{Reg}(N)}{w(N)} \quad$ où $w(N)$ est le nombre de racines de l'unité de $\mathbf{N}$,

$F_{N}(s)=A_{N}^{s} \Gamma\left(\frac{s}{2}\right)^{r_{1}} \Gamma(s)^{r_{2}} \zeta_{N}(s)$

de sorte que $F_{N}(s)$ a un pôle simple en $s=1$ de résidu $\lambda_{N}$.

Soit $K / k$ une extension de corps de nombres. Nous ne nous plaçons que dans des situations pour lesquelles la fonction $\Phi_{K / k} \stackrel{\text { def }}{=} \zeta_{K} / \zeta_{k}$ est holomorphe dans tout le plan complexe. Nous voulons un moyen de calcul numérique de la valeur $\Phi_{K / k}(1)$. Les applications que nous visons étant alors : 
(i). Par le choix $\mathbf{k}=\mathbf{Q}$, nous désirons pouvoir calculer le produit du régulateur $\operatorname{Reg}(K)$ par le nombre de classes d'idéaux $h(K)$ de $\mathbf{K}$, de sorte que le calcul de ce régulateur par d'autres méthodes nous donnera le nombre de classes de $\mathbf{K}$. Notons par exemple que si $k=Q$ et si $K=Q(\sqrt[p]{m}), m \geq 2$ est un corps pur de degré premier, alors $\Phi_{K / k}$ est bien holomorphe dans tout le plan complexe (et ce parce que $\left(\Phi_{K / Q}\right)^{p-1}=\Phi_{N / K}$ est holomorphe puisque $N / K$ est abélienne, où $N=Q\left(\zeta_{p}, \sqrt[p]{m}\right)$ est la clôture normale de $\left.\mathbf{K}\right)$.

(ii). Par le choix $\mathbf{k}=$ le sous-corps totalement réel maximal d'un corps à multiplication complexe $\mathbf{K}$, nous désirons pouvoir calculer le nombre de classes relatif $h^{*}(K)$ de $\mathbf{K}$.

Nous posons $\Psi_{K / k}=F_{K} / F_{k}$, de sorte que $s \mapsto \Psi_{K / k}(s)$ est invariante par $s \mapsto 1-s$ et holomorphe dans tout le plan complexe si $\Phi_{K / k}$ l'est. Remarquons que :

(a). Dans la situation (i) nous avons $\Psi_{K / k}(1)=\lambda_{K} / \lambda_{Q}=\lambda_{K}$

(b). Dans la situation (ii) nous avons :

$$
\Psi_{K / k}(1)=\frac{\lambda_{K}}{\lambda_{k}}=\frac{h^{*}(K)}{Q_{K} w(K)}
$$

où $Q_{K}=1$ ou 2 est un indice d'unités (voir [Wa, Th. 4.12]).

2. Expression de $\Psi_{K / k}(1)$ comme somme d'une série absolument convergente et à convergence rapide. Il s'agit de généraliser la méthode développée dans [BLW]. Définissons les coefficients $\phi_{n}$ par :

$$
\Phi_{K / k}(s)=\frac{\zeta_{K}}{\zeta_{k}}(s)=\sum_{n \geq 1} \phi_{n} n^{-s}, \Re(s)>1 .
$$

Notons que si $\mathbf{K}$ est un corps à multiplication complexe de sous-corps totalement réel maximal $\mathbf{k}$ et si $\chi_{K / k}$ est le caractère quadratique de l'extension quadratique $\mathbf{K} / \mathbf{k}$, alors $\left(\zeta_{K} / \zeta_{k}\right)(s)=L\left(s, \chi_{K / k}\right)$, de sorte que nous avons

$$
\phi_{n}=\sum_{N_{k / Q}(I)=n} \chi_{K / k}(I)
$$

où cette sommation porte sur les idéaux entiers $\mathbf{I}$ de norme $n$ du corps $\mathbf{k}$.

Puisque

$$
\Gamma(s)=\frac{2^{s-1}}{\sqrt{\pi}} \Gamma\left(\frac{s}{2}\right) \Gamma\left(\frac{s+1}{2}\right),
$$

pour $c_{K / k}, A_{K / k}$ des réels et pour $a, b$ et $c$ des entiers positifs ou nuls, nous pouvons écrire

$$
\Psi_{K / k}(s)=c_{K / k} A_{K / k}^{s} \Gamma\left(\frac{s}{2}\right)^{a} \Gamma\left(\frac{s+1}{2}\right)^{b} \Gamma(s)^{c} \Phi_{K / k}(s) .
$$


Notons que si $\mathbf{K}$ est un corps à multiplication complexe de degré $2 N$ de sous-corps totalement réel maximal $\mathbf{k}$ alors de degré $N$, nous avons :

$$
(a, b, c)=(0, N, 0), \quad A_{K / k}=\sqrt{\frac{d(K)}{\pi^{N} d(k)}} \text { et } \quad c_{K / k}=\frac{1}{(4 \pi)^{N / 2}} .
$$

Notons également que nous pourrions de plus imposer à $c$ d'être nul, ce qui permettrait de ne considérer des fonctions $H_{(d, e)}$ ne dépendant ue de deux indices. Si nous ne le faisons pas, c'est parce qu'il est maladroit de par exemple vouloir imposer $c=0$ puisque dans la situation $\mathbf{k}=\mathbf{Q}$ et $\mathbf{K}=\mathbf{u n}$ corps pur de degré premier $p$, cela impliquerait de faire intervenir la fonction $H_{((p-1) / 2,(p-1) / 2,0)}$ au lieu de la plus simple $H_{(0,0,(p-1) / 2)}$.

Définissons la transformée de Mellin $\hat{\Psi}_{K / k}$ de $\Psi_{K / k}$ :

$$
\hat{\Psi}_{K / k}(x)=\frac{1}{2 i \pi} \int_{\alpha-i \infty}^{\alpha+i \infty} \Psi_{K / k}(s) x^{-s} d s, \alpha>1
$$

de sorte que

$$
\hat{\Psi}_{K / k}(x)=c_{K / k} \sum_{n \geq 1} \phi_{n} H_{(a, b, c)}\left(\frac{n x}{A_{K / k}}\right), x>0
$$

où nous posons :

$$
H_{(a, b, c)}(x)=\frac{1}{2 i \pi} \int_{\alpha-i \infty}^{\alpha+i \infty} \Gamma\left(\frac{s}{2}\right)^{a} \Gamma\left(\frac{s+1}{2}\right)^{b} \Gamma(s)^{c} x^{-s} d s, x>0 \text { et } \alpha>0 .
$$

Puisque $\Psi_{K / k}(s)=\Psi_{K / k}(1-s)$ et puisque $\Psi_{K / k}$ est (supposée être) holomorphe dans tout le plan complexe, par déplacement parallèle de l'axe vertical d'intégration d'abscisse $\alpha$ jusqu'à l'axe vertical d'intégration d'abscisse $1-\alpha$, puis par utilisation de cette équation fonctionnelle pour revenir sur l'axe vertical d'intégration d'abscisse $\alpha$, nous en déduisons l'équation fonctionnelle suivante :

$$
x \hat{\Psi}_{K / k}(x)=\hat{\Psi}_{K / k}(1 / x), \quad x>0 .
$$

La formule d'inversion de Mellin implique alors :

$$
\Psi_{K / k}(s)=\int_{0}^{\infty} \hat{\Psi}_{K / k}(x) x^{s} \frac{d x}{x}=\int_{1}^{\infty} \hat{\Psi}_{K / k}(x)\left\{x^{s-1}+x^{-s}\right\} d x .
$$

Il en résulte :

$$
\Psi_{K / k}(1)=\int_{1}^{\infty} \hat{\Psi}_{K / k}(x) d x+\int_{1}^{\infty} \hat{\Psi}_{K / k}(x) \frac{d x}{x} .
$$


Pour utiliser cette formule (3), il reste encore à calculer les deux intégrales suivantes :

$$
I_{(a, b, c)}(A) \stackrel{\text { def }}{=} \int_{A}^{\infty} H_{(a, b, c)}(x) d x \quad \text { et } J_{(a, b, c)}(A) \stackrel{\text { def }}{=} \int_{A}^{\infty} H_{(a, b, c)}(x) \frac{d x}{x}
$$

qui en posant

$$
K_{(a, b, c)}(A) \stackrel{\text { def }}{=} I_{(a, b, c)}(A)+A J_{(a, b, c)}(A)
$$

donnent en tenant compte de (3) :

$$
\Psi_{K / k}(1)=c_{K / k} A_{K / k} \sum_{n \geq 1} \frac{\phi_{n}}{n} K_{(a, b, c)}\left(\frac{n}{A_{K / k}}\right) .
$$

Nous n'allons pas montrer en toute généralité que cette série est absolument convergente, à convergence rapide et parfaitement adaptée au calcul numérique de $\Psi_{K / k}(1)$, mais allons uniquement prouver ces résultats dans le cas qui nous intéresse le plus : celui où $\mathbf{K}$ est un corps à multiplication complexe de sous-corps totalement réel maximal $\mathbf{k}$.

3. Calcul numérique du nombre de classes relatif des corps à multiplication complexe. Dans la situation particulière où $\mathbf{K}$ est un corps à multiplication complexe de degré $2 N$ et de sous-corps totalement réel maximal $\mathbf{k}$, (5) s'écrit en tenant compte de (1) :

$$
h^{*}(K)=\frac{Q_{K} w_{K}}{(2 \pi)^{N}} \sqrt{\frac{d(K)}{d(k)}} \sum_{n \geq 1} \frac{\phi_{n}}{n} K_{N}\left(\frac{n}{A_{K / k}}\right),
$$

où nous avons posé $K_{N}=K_{(0, N, 0)}$. Le changement de variables $s \mapsto 2 s-$ 1 donne $H_{(0, N, 0)}(x)=2 x H_{(0,0, N)}\left(x^{2}\right)$, et une inversion d'intégrales dans (4) donne alors :

$$
K_{N}(A)=\frac{A^{2}}{2 i \pi} \int_{\alpha-i \infty}^{\alpha+i \infty} 2 \Gamma(s)^{N} A^{-2 s}\left(\frac{1}{2 s-1}+\frac{1}{2 s-2}\right) d s \quad(\alpha>1) .
$$

La proposition suivante assurera l'absolue convergence et la convergence rapide de la série (6) :

Proposition 1. Pour $x>0$ et $N \geq 1$, nous avons : (a). $0 \leq H_{(0,0, N)}(x) \leq 2^{N-1} e^{-x^{1 / N}} / x^{1-(1 / N)}$. 
(b). $0 \leq K_{N}(A) \leq N 2^{N} e^{-A^{2 / N}}, A>0$.

Preuve. Nous ne prouvons que ces majorations, et ce par récurrence sur $N$. En effet,

$$
H_{(0,0,1)}(x)=e^{-x} \quad \text { et } \quad H_{(0,0, N+1)}(x)=\int_{0}^{\infty} H_{(0,0, N)}(t) e^{-x / t} \frac{d t}{t} .
$$

En faisant le changement de variable $t \mapsto x^{N /(N+1)} T^{N}$, on en déduit par récurrence sur $N$

$$
H_{(0,0, N+1)}(x) \leq \frac{N 2^{N-1}}{x^{1-(2 /(N+1))}} \int_{0}^{\infty} \exp \left(-x^{1 /(N+1)}\left(T+\frac{1}{T^{N}}\right)\right) \frac{d T}{T^{N}} .
$$

En scindant cette intégrale entre 0 et l'infini en une somme d'une intégrale entre 1 et l'infini et une intégrale entre 0 et 1 , puis en faisant le changement de variable $T \mapsto 1 / T$ dans cette seconde intégrale nous obtenons :

$$
\begin{aligned}
H_{(0,0, N+1)}(x) \leq \frac{N 2^{N-1}}{x^{1-(2 /(N+1))}} & \left\{\int_{1}^{\infty} \exp \left(-x^{1 /(N+1)}\left(T+\frac{1}{T^{N}}\right)\right) \frac{d T}{T^{N}}\right. \\
& \left.+\int_{1}^{\infty} \exp \left(-x^{1 /(N+1)}\left(T^{N}+\frac{1}{T}\right)\right) T^{N-2} d T\right\} \\
\leq \frac{N 2^{N-1}}{x^{1-(2 /(N+1))}}\left\{\int_{1}^{\infty} \exp \left(-x^{1 /(N+1)} T\right) \frac{d T}{T^{N}}\right. & \\
& \left.+\int_{1}^{\infty} \exp \left(-x^{1 /(N+1)} T^{N}\right) T^{N-1} d T\right\}
\end{aligned}
$$

Pour $N \geq 0$ posons

$$
I_{N}(\alpha)=\int_{1}^{\infty} e^{-\alpha T} \frac{d T}{T^{N}}
$$

Nous avons pour $N \geq 1$ :

$$
I_{N}(\alpha) \leq I_{N-1}(\alpha)=\frac{e^{-\alpha}}{\alpha}-(N-1) I_{N}(\alpha)
$$

(intégration par parties), qui pour $N \geq 1$ implique

$$
I_{N}(\alpha) \leq \frac{e^{-\alpha}}{N \alpha}, \alpha>0
$$


D'où :

$$
H_{(0,0, N+1)}(x) \leq 2^{N} \frac{e^{-x^{1 /(N+1)}}}{x^{1-(1 /(N+1))}}
$$

et le (a) est prouvé.

La majoration de $K_{N}(A)$ résulte alors de

$$
\begin{aligned}
K_{N}(A) \leq 2 I_{(0, N, 0)}(A) & =2 \int_{A}^{\infty} 2 x H_{(0,0, N)}\left(x^{2}\right) d x \\
& =\int_{A^{2}}^{\infty} 2 H_{(0,0, N)}(x) d x \\
& \leq \int_{A^{2}}^{\infty} 2^{N} e^{-x^{1 / N}} x^{1 / N} \frac{d x}{x} \\
& =\int_{A^{2 / N}}^{\infty} N 2^{N} e^{-x} d x=N 2^{N} e^{-A^{2 / N}}
\end{aligned}
$$

Proposition 2. Pour $N \geq 2$ et $\lambda>1$ fixés, $\left|h^{*}(K)-h_{\text {approx }}^{*}(K)\right|$ tend vers 0 lorsque $d(K)$ tend vers l'infini, où $h_{\mathrm{approx}}^{*}(K)$ est le réel obtenu en stoppant la sommation de (6) aux indices $n$ majorés par $A_{K / k}\left(\lambda \log A_{K / k}\right)^{N / 2}$.

Preuve. Nous remarquons que $\left|\phi_{n}\right|$ est d'après (2) majoré par $d_{N}(n)$ où $d_{N}(n)$ désigne le nombre de factorisations de $n$ en produit de $N$ entiers strictement positifs. Nous remarquons ensuite que

$$
S_{N}(x)=\sum_{n \leq x} \frac{d_{N}(n)}{n} \leq\left(\sum_{n \leq x} 1 / n\right)^{N} \leq \log ^{N}(e x) .
$$

Lemme 3. Pour $M \geq 1$ entier vérifiant $M \geq A\left(N^{2} / 2\right)^{N / 2}$ nous avons

$$
R_{M}=\sum_{n>M} \frac{d_{N}(n)}{n} e^{-(n / A)^{2 / N}} \leq\left(\log (e M)+\left(N^{2} / 2\right)\right)^{N} e^{-(M / A)^{2 / N}} .
$$

Preuve du Lemme. Une transformation d'Abel donne

$$
\begin{aligned}
R_{M} & \leq \sum_{n>M} S_{N}(n)\left(e^{-(n / A)^{2 / N}}-e^{-((n+1) / A)^{2 / N}}\right) \\
& =\sum_{n>M} S_{N}(n) \int_{n}^{n+1} \frac{2}{A N}\left(\frac{u}{A}\right)^{(2 / N)-1} e^{-(u / A)^{2 / N}} d u \\
& \leq \frac{2}{A N} \sum_{n>M}\left(\frac{n}{A}\right)^{(2 / N)-1} e^{-(n / A)^{2 / N}} \log ^{N}(e n) .
\end{aligned}
$$


Posons $e A=\alpha^{N / 2}$ et remarquons que

$$
u \mapsto f(u)=\left(\frac{u}{A}\right)^{(2 / N)-1} e^{-(u / A)^{2 / N}} \log ^{N}(e u)
$$

est décroissante sur $\left[A\left(N^{2} / 2\right)^{N / 2},+\infty[\right.$. Nous obtenons :

$$
\begin{aligned}
R_{M} & \leq \frac{2}{N} \int_{M}^{\infty}(x / A)^{2 / N} e^{-(x / A)^{2 / N}} \log ^{N}(e x) \frac{d x}{x} \\
& =(N / 2)^{N} \int_{(M / A)^{2 / N}}^{\infty} e^{-U} \log ^{N}(\alpha U) d U \quad\left(\text { poser } x=A U^{N / 2}\right) .
\end{aligned}
$$

Maintenant, en posant $I_{N}(x)=\int_{x}^{\infty} \log ^{N}(\alpha U) e^{-U} d U$, nous avons $I_{0}(x)=e^{-x}$ et

$$
\begin{aligned}
I_{N}(x) & =e^{-x} \log ^{N}(\alpha x)+\int_{x}^{\infty} N \log ^{N-1}(\alpha x) e^{-U} \frac{d U}{U} \quad(N \geq 1) \\
& \leq e^{-x} \log ^{N}(\alpha x)+N I_{N-1}(x) \quad(\text { pour } x \geq 1)
\end{aligned}
$$

qui conduit par récurrence sur $N$ à la majoration $I_{N}(x) \leq(\log (\alpha x)+$ $N)^{N} e^{-x}$ pour $x \geq 1$ et $N \geq 0$. D'où le Lemme puisque $\alpha(M / A)^{2 / n}=$ $(e M)^{2 / N}$.

Fin de la preuve de la Proposition 2. D'après (6) et la Proposition 1, en posant $A=A_{K / k}$ (qui tend vers l'infini lorsque $d(K)$ tend vers l'infini puisque on a $\left.d(K) \geq(d(k))^{2}\right)$, nous obtenons :

$$
\frac{Q_{K} w_{K}}{(2 \pi)^{N}} \sqrt{\frac{d(K)}{d(k)}} \sum_{n>M} \frac{\left|\phi_{n}\right|}{n} K_{N}\left(\frac{n}{A_{K / k}}\right) \leq \frac{2 N w_{K}}{\pi^{N / 2}} A R_{M}
$$

qui pour $M \geq A(\lambda \log A)^{N / 2}$ donne d'après le Lemme 3 :

$$
\left|h^{*}(K)-h_{\text {approx }}^{*}(K)\right| \leq \frac{2 N w_{K}}{\pi^{N / 2}} \frac{\left(\log (e M)+\left(N^{2} / 2\right)\right)^{N}}{A^{\lambda-1}} .
$$

Remarque. Notons que si $\mathbf{K} / \mathbf{Q}$ est abélienne non quadratique de conducteur $f$ et à régulateur de taille petite par rapport au discriminant de $\mathbf{K}$, il est sans intérêt d'appliquer notre méthode puisque l'identité (5) nous dit qu'il nous faudrait sommer au moins de l'ordre de $A_{K / k}$ termes, donc de l'ordre 
de $f^{\frac{1}{2}([K: Q]-1)+\epsilon}$ termes, alors que le calcul du nombre de classes à partir des développements des fonctions $L$ au point 1 (voir [ScW] et [SeWW]) ne nécessite des sommations que de l'ordre de $f^{\frac{1}{2}+\epsilon}$ termes, et que son calcul à partir des expressions explicites de sommes finies de ces valeurs au point 1 ne nécessite que des sommations de l'ordre de $f$ termes.

\section{Calcul numérique des nombres de classes relatifs des corps quar-} tiques totalement imaginaires non galoisiens. Nous montrons sur cet exemple que la Proposition 2 est particulièrement bien adaptée au calcul des nombres de classes relatifs des corps à multiplication complexe $\mathbf{K}$. En particulier, notre méthode conduit à un calcul numérique des nombres de classes de ces corps nettement plus efficient que celui développé dans [Oka]. Nous venons de voir où arrêter la sommation de la série (6) de sorte que la somme finie ainsi définie conduise à une bonne approximation du nombre de classes relatif de $\mathbf{K}$. Nous expliquons maintenant comment déterminer une valeur numérique approchée de chacun des termes de la série (6) et expliquerons finalement sur un exemple comment calculer les valeurs numériques des coefficients $\phi_{n}$ (nous renvoyons à [Lou 2], [Lou 3] et [LOO] pour d'autres exemples de tels calculs).

Calcul numérique des fonctions intégrales apparaissant dans la formule analytique. Nous illustrons sur le cas particulier $N=2$ qui nous occupe maintenant une méthode générale de calcul des développements en séries entières des fonctions $K_{N}$ apparaissant à l'identité (6). On trouvera dans [Lou 3] un algorithme général de leur calcul valable pour tout $N \geq 1$. Noter une faute d'impression au Lemme de la page 322 de [Lou 3]. Il faut y lire

$$
=-(-1)^{N n} \frac{A^{2 n}}{(n !)^{N}} \quad\left(\text { au lieu de }=-\frac{A^{2 n}}{(n !)^{N}}\right) .
$$

Proposition 4. Pour $A>0$ et avec la convention $\sum_{k=1}^{0} \frac{1}{k}=0$, nous avons le développement suivant (où $\gamma=0.577215664901532 \cdots$ est la constante d'Euler) :

$$
\begin{aligned}
& K_{2}(A)=1+\pi A+4(\log (A)+\gamma) \sum_{n \geq 0}\left(\frac{1}{2 n+1}+\frac{1}{2 n+2}\right) \frac{A^{2 n+2}}{(n !)^{2}} \\
& -4 \sum_{n \geq 0}\left(\frac{1}{(2 n+1)^{2}}+\frac{1}{(2 n+2)^{2}}+\left(\frac{1}{2 n+1}+\frac{1}{2 n+2}\right) \sum_{k=1}^{n} \frac{1}{k}\right) \frac{A^{2 n+2}}{(n !)^{2}} .
\end{aligned}
$$

Preuve. On remarque que

$$
s \mapsto f(s)=2 \Gamma^{2}(s) A^{2-2 s}\left(\frac{1}{2 s-1}+\frac{1}{2 s-2}\right)
$$


possède un pôle simple en $s=1$ de résidu $\operatorname{Res}_{1}(f)=1$, possède un pôle simple en $s=\frac{1}{2}$ de résidu $\operatorname{Res}_{1 / 2}(f)=\pi A$, et possède un pôle double en chaque $s=-n,(n \geq 0)$ de résidu

$$
\begin{aligned}
& \operatorname{Res}_{-n}(f)=4 \frac{A^{2 n+2}}{(n !)^{2}}\left(\left(\frac{1}{2 n+1}+\frac{1}{2 n+2}\right)\right. \\
&\left.\cdot\left(\log (A)-\frac{\Gamma^{\prime}}{\Gamma}(n+1)\right)-\frac{1}{(2 n+1)^{2}}-\frac{1}{(2 n+2)^{2}}\right) .
\end{aligned}
$$

De (7) et par déplacement vers la gauche de l'axe d'intégration, nous en déduisons aisément

$$
K_{N}(A)=\frac{1}{2 i \pi} \int_{\alpha-i \infty}^{\alpha+i \infty} f(s) d s=\operatorname{Res}_{1}(f)+\operatorname{Res}_{1 / 2}(f)+\sum_{n \geq 0} \operatorname{Res}_{-n}(f) .
$$

Le résultat s'en déduit en remarquant que le produit infini de la fonction Gamma donne : $\frac{\Gamma^{\prime}}{\Gamma}(n+1)=-\gamma+\sum_{k=1}^{n} \frac{1}{k}$ pour $n \geq 0$.

Calcul des coefficients $\phi_{n}$. Puisque $n \mapsto \phi_{n}$ est multiplicative, elle est déterminée par ses valeurs sur les puissances des nombres premiers, valeurs que nous donnons maintenant.

(i). Si $l$ est inerte dans $k / Q$ et non ramifié dans $K / Q$, alors $\phi_{l^{k}}=\frac{1+(-1)^{k}}{2} \chi_{K / k}((l))^{k / 2}$.

(ii). Si $l$ est inerte dans $k / Q$ et ramifié dans $K / Q$, alors $\phi_{l^{k}}=0$.

(iii). Si $l$ est ramifié en $(l)=L^{2}$ dans $k / Q$, alors $\phi_{l^{k}}=\chi_{K / k}(L)^{k}$.

(iv). Si $l$ est totalement décomposé en $(l)=L L^{\prime}$ dans $k / Q$ et non ramifié dans $K / Q$, alors

$$
\begin{aligned}
\phi_{l^{k}} & =\sum_{i=0}^{k} \chi_{K / k}\left(L^{k-i} L^{\prime i}\right) \\
& =\chi_{K / k}(L)^{k} \sum_{i=0}^{k} \chi_{K / k}\left(L L^{\prime}\right)^{i} \\
& = \begin{cases}(k+1) \chi_{K / k}(L)^{k} & \text { si } \chi_{K / k}\left(\mathbf{L} \mathbf{L}^{\prime}\right)=1, \\
\frac{1+(-1)^{k}}{2} & \text { si } \chi_{K / k}(\mathbf{L L})=-1 .\end{cases}
\end{aligned}
$$


(v). Si $l$ est totalement décomposé en $(l)=L L^{\prime}$ dans $k / Q$ et ramifié dans $K / Q$, de sorte que l'on peut supposer $\mathbf{L}$ ramifié dans $K / k$, alors $\phi_{l^{k}}=$ $\chi_{K / k}\left(L^{\prime}\right)^{k}$.

Nous nous plaçons maintenant dans la situation considérée dans [Lou 4] et $[\mathbf{L O}]$, et expliquons comment nous avons calculé les nombres de classes relatifs des corps quartiques à multiplication complexe qui apparaissent dans ces deux articles. Soit donc $p \equiv 1(\bmod 4)$ premier tel que $k=Q(\sqrt{p})$ soit principal. Nous nous intéressons maintenant uniquement aux corps quartiques non galoisiens totalement imaginaires $\mathbf{K}$ de sous-corps quadratique réel $\mathbf{k}$ et de discriminants relatifs premiers dans $\mathbf{k}$. Il est aisé de voir que ce sont précisément les $K_{(p, q)}=k\left(\sqrt{-\alpha_{q}}\right)$ où $\alpha_{q}=\left(x_{q}+y_{q} \sqrt{p}\right) / 2$ un entier algébrique totalement positif, primaire (c'est à dire congru à un carré modulo l'idéal $(4)$ de $\mathbf{k})$ et de norme $q$ avec $q \equiv 1(\bmod 4)$ un nombre premier donc totalement décomposé dans $\mathbf{k}$. Imposer à un tel $\alpha_{q}$ d'être primaire est équivalent à demander que l'on ait $x_{q} \equiv 1(\bmod 4)$ lorsque $x_{q}$ est impair, et $x_{q} \equiv y_{q}+6(\bmod 8)$ lorsque $x_{q}$ est pair. Notons que $K_{(p, q)}$ est de discriminant $d\left(K_{(p, q)}\right)=p^{2} q$.

Si $L=(\lambda)$ est un idéal premier principal de $\mathbf{k}$, où $\lambda$ est choisi totalement positif (ce qui est toujours possible), d'après les lois de réciprocité (voir $[\mathbf{H e c}])$, nous avons :

$$
\chi_{K / k}(L)=\left[\frac{-\alpha_{q}}{L}\right]=\left[\frac{\lambda}{\left(\alpha_{q}\right)}\right]
$$

Puisque $\left(\alpha_{q}\right)$ est un idéal premier totalement décomposé de $\mathbf{k}$ de norme $q$ donc première et congrue à 1 modulo 4 , les résultats de [Lou 1] (plus précisément, [Lou 1, Th. 5(b)]) dont la validité reste acquise dans ce cadre donnent :

$$
\left[\frac{Z}{\left(\alpha_{q}\right)}\right]=\left(\frac{T r_{k / Q}\left(\alpha_{q}\right) T r_{k / Q}\left(\alpha_{q} Z^{\prime}\right)}{q}\right)
$$

où $Z^{\prime}$ désigne le conjugué dans $\mathbf{k}$ d'un entier algébrique $Z$ de $\mathbf{k}$, où $T r_{k / Q}(Z)=$ $Z+Z^{\prime}$ désigne la trace de $z$, et où $(\bullet / q)$ désigne le symbole de Legendre. D'où :

(vi). $\chi_{K / k}(L)=(l / q)$ lorsque $L=(l)$ est premier inerte dans $\mathbf{k}$.

(vii). $\chi_{K / k}(L)=\left(2 x_{q}\left(x_{\lambda} x_{q}-p y_{\lambda} y_{q}\right) / q\right)=\left(2\left(x_{\lambda} y_{q}-y_{\lambda} x_{q}\right) / q\right)\left(\operatorname{car} x_{q}^{2}=p y_{q}^{2}+\right.$ $4 q$ et $(p / q)=+1$ et $\left.\left(y_{q} / q\right)=+1\right)$ lorsque $L=(\lambda)$ avec $l=\lambda \lambda^{\prime}$ premier non 
inerte dans $\mathbf{k}$ et avec $\lambda=\left(x_{\lambda}+y_{\lambda} \sqrt{d}\right) / 2$ totalement positif. De plus, d'après (9) nous avons alors:

$$
\chi_{K / k}\left(L L^{\prime}\right)=\left[\frac{\lambda}{(\alpha)}\right]\left[\frac{\lambda^{\prime}}{(\alpha)}\right]=\left[\frac{\lambda \lambda^{\prime}}{(\alpha)}\right]=\left[\frac{l}{(\alpha)}\right]=\left(\frac{l}{q}\right)
$$

Nous avons alors les formules complètement explicites suivantes :

(viii). Si $l \neq p, q$ est inerte dans $\mathbf{k}$, alors $\phi_{l^{k}}=\frac{1+(-1)^{k}}{2}\left(\frac{l}{q}\right)^{k / 2}$,

(ix). Si $l \neq p, q$ est totalement décomposé en $(l)=L L^{\prime}$ dans $\mathbf{k}$ et si $L=(\lambda)$ où $\lambda=\left(x_{\lambda}+y_{\lambda} \sqrt{p}\right) / 2$ avec $x_{\lambda} \geq 1$ et $y_{\lambda} \geq 1$ tels que $x_{\lambda}^{2}-p y_{\lambda}^{2}=4 l$ alors

$\chi_{K / k}(L)=\left(\frac{2\left(x_{\lambda} y_{q}-y_{\lambda} x_{q}\right)}{q}\right) \quad$ et $\phi_{l^{k}}= \begin{cases}(k+1) \chi_{K / k}(\mathbf{L})^{k} & \text { si }(l / q)=1, \\ \frac{1+(-1)^{k}}{2} & \text { si }(l / q)=-1 .\end{cases}$

(x). Si $l=q$, alors $\phi_{q^{k}}=\left(\frac{x_{q}}{q}\right)^{k}$

$\left(\operatorname{car} \chi_{K / k}\left(Q^{\prime}\right)=\left(4 x_{q} y_{q} / q\right)=\left(x_{q} / q\right)\right.$ puisque $Q^{\prime}=(\lambda)$ avec $\lambda=\left(x_{q}-\right.$ $\left.\left.y_{q} \sqrt{p}\right) / 2\right)$.

(xi). Si $l=p$, alors $\phi_{p^{k}}=\left(\frac{2 x_{q}}{p}\right)^{k}$

$\left(\operatorname{car} \chi_{K / k}(P)=\left[-\alpha_{q} /(\sqrt{p})\right]=\left[-4 \alpha_{q} /(\sqrt{p})\right]=\left[-2 x_{q} /(\sqrt{p})\right]=\left(-2 x_{q} / p\right)=\right.$ $\left(2 x_{q} / p\right)$ puisque $-4 \alpha_{q}=-2 x_{q}-2 y_{q} \sqrt{p} \equiv 2 x_{q}(\bmod P)$. On peut de plus montrer que $\left.\left(2 x_{q} / p\right)=-\left(x_{q} / q\right)\right)$.

Nous donnons au tableau suivant les nombres de classes relatifs de tous les $K_{(p, q)}$ possibles tels que $5 \leq p<q<229$ (noter que 229 est le plus petit nombre premier $p \equiv 1(\bmod 4)$ tel que le corps quadratique réel $Q(\sqrt{p})$ ne soit pas principal). Notons que l'on peut montrer (voir [LO]) que $h^{*}\left(K_{(p, q)}\right)=h^{*}\left(K_{(q, p)}\right)$. Nous avons utilisé cette relation comme moyen de vérification de la justesse de l'implémentation de notre méthode, mais limitons évidemment notre tableau de valeurs de ces nombres de classes relatifs aux couples $(p, q)$ tels que $p<q$. 


\begin{tabular}{|cc|cc|cc|}
\hline$(p, q)$ & $h^{*}\left(K_{(p, q)}\right)$ & $(p, q)$ & $h^{*}\left(K_{(p, q)}\right)$ & $(p, q)$ & $h^{*}\left(K_{(p, q)}\right)$ \\
\hline$(5,41)$ & 1 & $(37,181)$ & 7 & $(101,181)$ & 9 \\
$(5,61)$ & 1 & $(41,73)$ & 11 & $(101,193)$ & 11 \\
$(5,109)$ & 1 & $(41,113)$ & 13 & $(101,197)$ & 5 \\
$(5,149)$ & 1 & $(53,89)$ & 5 & $(109,113)$ & 11 \\
$(13,17)$ & 1 & $(53,97)$ & 5 & $(109,173)$ & 7 \\
$(13,29)$ & 1 & $(53,113)$ & 5 & $(109,193)$ & 5 \\
$(13,113)$ & 3 & $(53,197)$ & 5 & $(113,157)$ & 11 \\
$(13,157)$ & 1 & $(61,73)$ & 3 & $(113,173)$ & 7 \\
$(13,181)$ & 1 & $(61,97)$ & 11 & $(137,193)$ & 29 \\
$(17,89)$ & 7 & $(61,137)$ & 7 & $(137,197)$ & 15 \\
$(17,101)$ & 3 & $(61,197)$ & 5 & $(149,173)$ & 7 \\
$(17,137)$ & 1 & $(73,97)$ & 1 & $(157,173)$ & 7 \\
$(29,53)$ & 1 & $(73,149)$ & 9 & $(157,193)$ & 7 \\
$(29,149)$ & 5 & $(73,181)$ & 17 & $(157,197)$ & 9 \\
$(29,173)$ & 3 & $(89,97)$ & 3 & $(181,193)$ & 29 \\
$(37,41)$ & 3 & $(89,109)$ & 11 & $(181,197)$ & 11 \\
$(37,53)$ & 3 & $(89,157)$ & 9 & & \\
$(37,73)$ & 7 & $(97,109)$ & 15 & & \\
$(37,101)$ & 3 & $(97,193)$ & 7 & & \\
$(37,137)$ & 5 & $(101,137)$ & 9 & & \\
\hline
\end{tabular}

Nous concluons cet article en donnant, pour $k=Q(\sqrt{p})$ fixé, la distribution des nombres de classes relatifs des 1500 premières extensions quadratiques de $\mathbf{k}$ du type précédent. Dans ce tableau, $c_{l}$ désignent le nombre de nombres de classes relatifs divisibles par $l$ (premier) et $S$ désigne la somme de ces nombres de classes relatifs.

\begin{tabular}{|c|c|c|c|c|c|c|c|c|}
\hline$Q(\sqrt{p})$ & $c_{3}$ & $c_{5}$ & $c_{7}$ & $c_{11}$ & $c_{13}$ & $c_{17}$ & $q_{\max }$ & $\mathrm{S}$ \\
\hline$Q(\sqrt{5})$ & 581 & 339 & 263 & 140 & 131 & 115 & 129001 & 45608 \\
\hline$Q(\sqrt{13})$ & 606 & 328 & 253 & 143 & 114 & 99 & 129061 & 87654 \\
\hline$Q(\sqrt{17})$ & 594 & 360 & 209 & 139 & 115 & 94 & 127649 & 133836 \\
\hline$Q(\sqrt{29})$ & 599 & 344 & 258 & 136 & 128 & 93 & 128941 & 117444 \\
\hline$Q(\sqrt{37})$ & 614 & 337 & 232 & 142 & 157 & 99 & 129097 & 155192 \\
\hline$Q(\sqrt{41})$ & 595 & 368 & 236 & 135 & 133 & 101 & 129769 & 223394 \\
\hline
\end{tabular}

Notons que pour les 1500 premiers corps quadratiques imaginaires de discriminant $-q \equiv 1(\bmod 4)$ premier nous avons les distributions suivantes de nombres de classes: 


\begin{tabular}{|c|c|c|c|c|c|c|c|}
\hline$c_{3}$ & $c_{5}$ & $c_{7}$ & $c_{11}$ & $c_{13}$ & $c_{17}$ & $q_{\max }$ & $\mathrm{S}$ \\
\hline 590 & 339 & 238 & 133 & 127 & 94 & 27367 & 81624 \\
\hline
\end{tabular}

Il est intéressant de relier ces résultats numériques aux heuristiques de CohenLenstra-Martinet ainsi qu'aux résultats de B.A. Datskovsky.

\section{References}

[BLW] P. Barrucand, J. Loxton and H.C. Williams, Some explicit upper bounds on the class number and regulator of a cubic field with negative discriminant, Pacific J. Math., 128 (1987), 209-222.

[CM] H. Cohen and J. Martinet, Class groups of number fields : numerical heuristics, Math. Comp., 177 (1987), 123-137.

[D] B.A. Datskovsky, A mean-value theorem for class numbers of quadratic extensions, Contemporary Mathematics, 143 (1993), 179-272.

[Hec] E. Hecke, Lectures on the Theory of Algebraic Numbers, Grad. Texts Math., 77, Springer-Verlag.

[Lou 1] S. Louboutin, L-functions and class numbers of imaginary quadratic fields and of quadratic extensions of an imaginary quadratic field, Math. Comp., 59 (1992), 213-230.

[Lou 2] S. Louboutin, Calcul des nombres de classes relatifs : application aux corps octiques quaternioniques à multiplication complexe, C. R. Acad. Sci. Paris, 317 (1993), 643646.

[Lou 3] S. Louboutin, Calcul des nombres de classes relatifs de certains corps de classes de Hilbert, C. R. Acad. Sci. Paris, 319 (1994), 321-325.

[Lou 4] S. Louboutin, On the class number one problem for non-normal quartic CM-fields, Tôhoku Math. J., 46 (1994), 1-12.

[LO] S. Louboutin and R. Okazaki, Determination of all non-normal quartic CM-fields and of all non-abelian normal octic CM-fields with class number one, Acta Arith., 67 (1994), 47-62.

[LOO] S. Louboutin, R. Okazaki et M. Olivier, The class-number one problem for some non-abelian normal CM-fields, preprint Université de Caen (1995).

[Oka] R. Okazaki, On evaluation of L-functions over real quadratic fields, J. Math. Kyoto Univ., 31 (1991), 1125-1153.

[ScW] R. Schoof and L.C. Washington, Quintic polynomials and real cyclotomic fields with large class numbers, Math. Comp., 182 (1988), 543-556.

[SeWW] E. Seah, L.C. Washington and H.C. Williams, The calculation of a large cubic class number with an application to real cyclotomic fields, Math. Comp., 163 (1983), 303-305.

[Wa] L. C. Washington, Introduction to Cyclotomic Fields, Grad. Texts Math., 83, Springer-Verlag.

Received November 17, 1992 and revised June 2, 1993.

Université de Caen, U.F.R. Sciences

Esplanade de la Paix

14032 CAFN CEDEY FRANCF 



\title{
PACIFIC JOURNAL OF MATHEMATICS
}

Founded by E. F. Beckenbach (1906-1982) and F. Wolf (1904-1989)

\section{EDITORS}

\author{
Sun-Yung Alice Chang (Managing Editor) \\ University of California \\ Los Angeles, CA 90095-1555 \\ pacific@math.ucla.edu
}

\section{F. Michael Christ}

University of California

Los Angeles, CA 90095-1555

christ@math.ucla.edu

Thomas Enright

University of California

San Diego, La Jolla, CA 92093

tenright@ucsd.edu

Nicholas Ercolani

University of Arizona

Tucson, AZ 85721

ercolani@math.arizona.edu
Robert Finn

Stanford University

Stanford, CA 94305

finn@gauss.stanford.edu

Vaughan F. R. Jones

University of California

Berkeley, CA 94720

vfr@math.berkeley.edu

Steven Kerckhoff

Stanford University

Stanford, CA 94305

spk@gauss.stanford.edu
Martin Scharlemann

University of California

Santa Barbara, CA 93106

mgscharl@math.ucsb.edu

Gang Tian

Courant Institute

New York University

New York, NY 10012-1100

tiang@taotao.cims.nyu.edu

V. S. Varadarajan

University of California

Los Angeles, CA 90095-1555

vsv@math.ucla.edu

\section{SUPPORTING INSTITUTIONS}

\section{CALIFORNIA INSTITUTE OF TECHNOLOGY \\ NEW MEXICO STATE UNIVERSITY \\ OREGON STATE UNIVERSITY \\ STANFORD UNIVERSITY \\ UNIVERSITY OF ARIZONA \\ UNIVERSITY OF BRITISH COLUMBIA \\ UNIVERSITY OF CALIFORNIA UNIVERSITY OF HAWAII}

\author{
UNIVERSITY OF MONTANA \\ UNIVERSITY OF NEVADA, RENO \\ UNIVERSITY OF OREGON \\ UNIVERSITY OF SOUTHERN CALIFORNIA \\ UNIVERSITY OF UTAH \\ UNIVERSITY OF WASHINGTON \\ WASHINGTON STATE UNIVERSITY
}

The supporting Institutions listed above contribute to the cost of publication of this Journal, but they are not owners or publishers and have no responsibility for its contents or policies.

Manuscripts must be prepared in accordance with the instructions provided on the inside back cover.

The Pacific Journal of Mathematics (ISSN 0030-8730) is published monthly except for July and August. Regular subscription rate: $\$ 215.00$ a year (10 issues). Special rate: $\$ 108.00$ a year to individual members of supporting institutions.

Subscriptions, orders for back issues published within the last three years, and changes of subscribers address should be sent to Pacific Journal of Mathematics, P.O. Box 4163, Berkeley, CA 94704-0163, U.S.A. Prior back issues are obtainable from Kraus Periodicals Co., Route 100, Millwood, NY 10546.

The Pacific Journal of Mathematics at the University of California, c/o Department of Mathematics, 981 Evans Hall, Berkeley, CA 94720 (ISSN 0030-8730) is published monthly except for July and August. Second-class postage paid at Berkeley, CA 94704, and additional mailing offices. POSTMASTER: send address changes to Pacific Journal of Mathematics, P.O. Box 6143, Berkeley, CA 94704-0163.

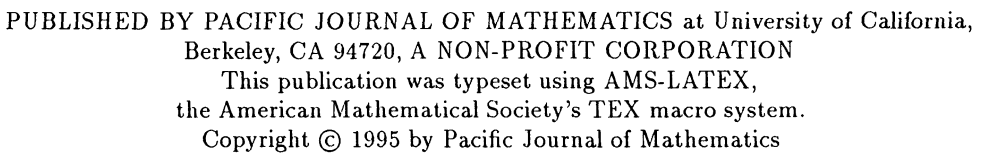




\section{PACIFIC JOURNAL OF MATHEMATICS}

\section{Volume $171 \quad$ No. $2 \quad$ December 1995}

On $H^{p}$-solutions of the Bezout equation

ERIC AMAR, JOAQUIM BRUNA FLORIS and ARTUR NiCOLAU

Amenable correspondences and approximation properties for von Neumann algebras

309

Claire ANANTHARAman-DElaroche

On moduli of instanton bundles on $\mathbb{P}^{2 n+1}$

VinCENZO ANCONA and Giorgio MaRia OtTAVIANI

Minimal surfaces with catenoid ends

JORGEN BERGLUND and WAYNE ROSSMAN

Permutation model for semi-circular systems and quantum random walks

PHILIPPE BIANE

The Neumann problem on Lipschitz domains in Hardy spaces of order less than one

RUSSELL M. BROWN

Matching theorems for twisted orbital integrals

409

REBECCA A. HERB

Uniform algebras generated by holomorphic and pluriharmonic functions on strictly

pseudoconvex domains

ALEXANDER IzZO

Quantum Weyl algebras and deformations of $U(g)$

NAIHUAN JING and JAMES ZHANG

Calcul du nombre de classes des corps de nombres

STÉPHANE LOUBOUTIN

On geometric properties of harmonic Lip ${ }_{1}$-capacity

PertTi MatTila and P. V. PARAmonov

Reproducing kernels and composition series for spaces of vector-valued holomorphic functions

Bent ØRsted and Genkai Zhang

Iterated loop modules and a filtration for vertex representation of toroidal Lie algebras

S. ESWARA RAO

The intrinsic mountain pass

MARTIN SCHECHTER

A Frobenius problem on the knot space

RON G. WANG

On complete metrics of nonnegative curvature on 2-plane bundles

DAVID YANG

Correction to: "Free Banach-Lie algebras, couniversal Banach-Lie groups, and more"

VLADIMIR G. PESTOV

Correction to: "Asymptotic radial symmetry for solutions of $\Delta u+e^{u}=0$ in a punctured disc"

KAI Seng (KAISing) CHOU (Tso) and Tom YAU-Heng WAN 